\title{
ACOMPANHAMENTO DA PRESSÃO ARTERIAL: estudo com crianças e adolescentes com história familiar de hipertensãoa
}

\author{
E milia Soares CHAVE Sb, Thelma L eite de ARAU J ${ }^{c}$, T ahissa Frota CAVALCAN TEd, \\ Nirla Gomes GUEDE $S^{d}$, R afaella Pessoa M OREIRAe
}

\section{RESUMO}

O bjetivou-se anal isar a evolução dos percentis de pressão arterial em crianças e adolescentes com história familiar de hipertensão arterial. E studo longitudinal desenvolvido em uma comunidade de Fortal eza, Ceará. A pressão arterial foi avaliada em cinco encontros domiciliares, de janeiro de 2004 a dezembro de 2006. G rupo de 141 participantes, dos quais 92 crianças e 49 adolescentes. D as crianças, 67,4\% per maneceram sem alterações dos percentis de pressão arterial em todas as avaliações; dos adolescentes, 65,3\% permaneceram nesta condição. A maior parte dos indivíduos mantiveram seus valores e percentis de pressões arteriais sistólica e diastólica el evados, e muitos que não apresentavam inicialmente alterações passaram a mostrálas no decorrer do acompanhamento. Confirma-se a necessidade de monitoração da pressão arterial rotineira de crianças e adolescentes tanto para a detecção precoce como para a prevenção de complicações.

D escritores: Saúde da criança. Saúde do adolescente. H ipertensão.

\section{RESUMEN}

E I objetivo fue analizar la evolución de los percentiles de presión arterial en niños y adolescentes con historia familiar de hipertensión arterial. E studio longitudinal en una comunidad de F ortaleza, Ceará, B rasil. L a presión arterial fue evaluada en cinco visitas domiciliares, de enero de 2004 a diciembre de 2006. E I grupo fue de 141 participantes, de los cuales había 92 niños y 49 adol escentes. D e entre los niños, 67,4\% permanecieron sin al teraciones de los percentiles de presión arterial en todas las evaluaciones; de entre los adol escentes, 65,3\% permanecier on en esta condición. L a mayor parte de los individuos mantuvier on sus valores y percentiles de presión arterial sistólica y presión arterial diastólica muy altos, y muchos que no presentaban inicialmente alteraciones, pasaron a señalarlas en el correr del acompañamiento. Así, se comprueba la necesidad de monitoración de la presión arterial rutinera de niños y adolescentes tanto para la detección como para la prevención de complicaciones.

Descriptores: Salud del niño. Salud del adolescente. H ipertensión.

T ítulo: A compañamiento de la presión arterial: investigación con niños y adol escentes con historia familiar de hipertensión.

\section{ABST RACT}

T he purpose of this study is to analyz ethe evolution of the percentiles of blood pressur ein children and adolescents with familial history of arterial hypertension. T his is a longitudinal study developed in a community in Fortaleza, Ceará, B razil. T he blood pressure was evaluated in five home visits from J anuary, 2004 to D ecember, 2006. T he group was composed of 141 participants, of which 92 were children and 49 adolescents. of the children, $67,4 \%$ remained with no alterations of the percentiles of bl ood pressurein all the evaluations; of the adol escents, $65,3 \%$ remained in the same condition. M ost individuals kept their values and percentiles of systolic blood pressureand diastolic blood pressure, and many that did not initially present alterations started to show some during the follow up. As a result, routine monitoring of blood pressure of children and adol escents proves necessar y for the early detection and prevention of complications.

Descriptors: Child health. A dolescent health. H ypertension.

T itle: B lood pressure tracking: study with children and adolescents with familial history of hypertension.

\footnotetext{
a Parte da tese de D outorado apresentada em 2007 ao D epartamento de Enfermagem da U niversidade Federal do Ceará (UFC).

${ }^{b}$ D outora em Enfermagem, Professora A djunto I do D epartamento de Enfermagem da U niversidade Federal do M aranhão (UFM A), São Luís, M aranhão, Brasil.

‘ D outora em Enfermagem, Professora Associada do D epartamento de Enfermagem da U FC, Coordenadora do Projeto Ações de Cuidado em Saúde Cardiovascular, Fortaleza, Ceará, Brasil.

d M estre em Enfermagem, D outoranda do Curso de Pós-G raduação em E nfer magem da U FC, Professora da F aculdade Católica Rainha do Sertão, Fortal eza, Ceará, Brasil.

e M estre em Enfermagem, D outoranda do Curso de Pós-G raduação em Enfermagem da U F C, Fortaleza, Ceará, Brasil.
} 


\section{INT RODUÇÃO}

A hipertensão arterial atinge grande parte da população em todo o mundo e se manifesta como uma síndrome caracterizada pela presença de níveis de pressão arterial elevados, associados a alterações hor monais e, no metabolismo, a fenômenos tróficos. Sua prevalência em crianças e adolescentes varia entre $1,2 \%$ e $13 \%$, na dependência de achados em estudos variados ${ }^{(1)}$.

A presença das alterações da pressão arterial em crianças e adolescentes tem evidenciado que a hipertensão pode ter sua história inicial nesta etapa de vida. No entanto, a atenção dos estudos se prende muito mais aos grupos de adultos e idosos. Confor me se acredita, os efeitos deletérios, no caso destas alterações, poderiam ser minimizados se a doença fosse detectada precocemente(2). Para tanto, bastaria avaliar periodicamente a pressão arterial e incluir esta responsabilidade como parte obrigatória das consultas às crianças e aos adolescentes.

A medida da pressão arterial é um procedimento usado tanto para o diagnóstico da hipertensão, como para o acompanhamento dos pacientes hipertensos e aval iação da eficácia terapêutica. D essa forma, esse procedimento é considerado de grande importância, pois níveis elevados de pressão arterial são tidos como um dos principais fatores de risco para morbidade e mortalidade cardiovasculares ${ }^{(2)}$.

De modo geral, a hipertensão arterial, detectada em indivíduos jovens, tem maior possibilidade de ser de origem secundária, decorrente de doenças renais, cardiovasculares, endocrinológicas, neurológicas e iatrogênicas. Entretanto, desde a década de 1970, observou-se que a hipertensão primária ou essencial, da qual não se conhece a origem, vem aumentando nas faixas etárias mais bai$\mathrm{xas}^{(3)}$. Outros autores consideram que escolares e, em particular, adolescentes, podem apresentar hipertensão primária ou essencial, usualmente detectada pela avaliação rotineira da pressão arterial(4).

D esta forma, a opinião convencional, segundo a qual a hiper tensão na criança é um evento raro e, na maioria das vezes, secundário a outra doença, tem sido questionada por estudos epidemiológicos que têm mostrado aumento da prevalência de hipertensão essencial na faixa etária pediátrica ${ }^{(3)}$.

Além disso, conforme revelam esses estudos sobre pressão arterial na infância, a existência de valores el evados nesta fase da vida reforça a hipótese de que a hipertensão em adultos é resultado direto de hipertensão na infância(5). T al afirmação também é mencionada em alguns estudos epidemiológicos internacionais sobre pressão arterial em crianças e adol escentes, mostrando que sua elevação nesses indivíduos é fator preditor de continuidade das el evações da pressão e do aumento do risco cardiovascular na vida adulta(6).

Prevenir o aumento da pressão arterial, al ém dos limites de normalidade, constitui maneira eficiente de combater a hipertensão, evitando o elevado custo social do seu tratamento e suas complicações $^{(1)}$.

Alguns estudos mostram a importância do fator familiar na etiologia da hipertensão arterial. E $m$ trabal ho realizado em uma escola de ensino fundamental e médio da cidade de Fortaleza, Ceará, os antecedentes familiares para a hipertensão arterial foram identificados em $55,6 \%$ dos indivíduos avaliados. A presença do indicador de risco história familiar de hipertensão determina maior risco para que os filhos também desenvolvam hipertensão arterial ${ }^{(7)}$.

Diante desta realidade, este estudo teve como propósito acompanhar a evolução de valores e percentis de pressão arterial de crianças e adolescentes com história familiar de hipertensão arterial.

\section{METODOLOGIA}

Tratou-se de um estudo longitudinal/ prospectivo. Como estudos prospectivos, entendem-se aqueles nos quais pessoas com uma característica ou um atributo específico são identificadas e observadas por um período de tempo para verificar se houve ou não um resultado ou condição de interesse ${ }^{(8)}$. E ste trabal ho originou-se de uma T ese, com parte de seus dados apresentados neste arti$\mathrm{go}^{(9)}$.

A pesquisa foi desenvolvida em uma das áreas de atendimento da E stratégia Saúde da F amília de deter minado bairro do município de F ortaleza, Ceará. Seus participantes foram crianças e adolescentes com familiares acompanhados pelo programa de tratamento de hipertensão arterial. A amostra inicial do estudo foi constituída por crianças e adolescentes (6 a 18 anos) integrantes das famílias atendidas na área de abrangência da U nidade Básica de Saúde da Família (U BASF ). 
A o longo do estudo, al guns participantes mudaram de domicílio e deixaram de fazer parte da área de abrangência de atendimento da UBASF. Inicialmente, tinham-se 163 participantes. Ao final da quinta avaliação, a amostra foi reduzida a 141 participantes, com uma perda de $13,5 \%$. A perda ocorrida deveu-se à mudança de domicílio para outros estados ou para outro local não identificado.

Inicial mente, foi real izado o levantamento dos indivíduos com al ter ações da pressão arterial, considerado neste estudo como primeiro acompanhamento, ocorrido no primeiro semestre de 2004. A partir dos resultados obtidos neste primeiro momento, consolidado em uma dissertação de M estrado(10), o grupo continuou a ser acompanhado. Foi proposto, de início, o acompanhamento por um período de trinta e seis meses, com avaliações, em média, a cada seis meses, no total de seis observações. No entanto, em virtude do limite máximo de perdas aceitáveis, que equival ea $15 \%{ }^{(11)}$, houve mais quatro encontros: primeiro semestre de 2005; segundo semestre de 2005; primeiro semestre de 2006 e segundo semestre de 2006.

Os critérios de inclusão foram estabelecidos na primeira etapa do estudo: ter idade entre 6 anos e 18 anos; ser familiar de pessoa portadora do diagnóstico de hipertensão arterial; residir na área de abrangência de atendimento da U nidade Básica de Saúde; aceitação dos pais ou responsáveis legais para participação no estudo e o respectivo aceite por parte das crianças e adolescentes.

A coleta de dados, realizada em domicílio, constou do registro da pressão arterial, verificada duas vezes com trinta segundos de intervalo entre as medidas; do peso, da estatura (para avaliação do índice de massa corporal) e da circunferência do braço da criança para escolha do manguito a ser utilizado. A escolha do manguito era feita após a medida da circunferência do braço das crianças e dos adolescentes com fita métrica não distensível, no ponto médio entre 0 acrômio e o olécrano. $\mathrm{Na}$ verificação das pressões arteriais, foram usados esfigmomanômetro aneróide com manguitos de largur as de $6 \mathrm{~cm}, 6,5 \mathrm{~cm}, 8 \mathrm{~cm}, 9 \mathrm{~cm}, 10 \mathrm{~cm}, 11 \mathrm{~cm}$, $12 \mathrm{~cm}$ e $13 \mathrm{~cm}$, e estetoscópio biauricular, sendo utilizado o diafragma para a audição dos sons. 0 manguito escolhido foi o de largura mais próxima correspondente a $40 \%$ da circunferência do braço do indivíduo ${ }^{(1)}$.

0 ambiente das coletas era tranqüilo, com 0 participante em posição sentada por cinco minu- tos antes da medida, pés apoiados no chão, braço estendido na altura do quarto espaço intercostal e sob superfície plana e sólida.

Para a tomada das medidas da pressão arterial, foram fontes obrigatórias de referência, os trabal hos da A merican $\mathrm{H}$ eart A ssociation ${ }^{(12)}$. As medidas foram realizadas pelos pesquisadores os quais padronizaram o modo de verificar conforme o referencial já mencionado.

A inter pretação dos valores pressóricos das crianças e adolescentes foi realizada usando-se, como referência, a classificação estabel ecida pelas V Diretrizes Brasileiras de Hipertensão Arterial, que utiliza como parâmetros tabelas de percentis. Conforme esta classificação, a pressão arterial pode ser: normal (menor que o percentil 90); limítr ofe (igual ao percentil 90 e menor que 95); hipertensão/ acima (maior que o percentil 95)(1). A demais, foram levados em conta, neste estudo, tanto os percentis isolados de pressão arterial sistólica (PAS) e de pressão arterial diastólica (PAD ), como em conjunto.

Ilustrativamente, os dados estão apresentados em forma de tabelas e gráficos e discutidos de acordo com a literatura pertinente. $\mathrm{Na}$ distribuição dos participantes, crianças e adolescentes, levou-se em consider ação a idade informada por ocasião do início do estudo. Os resultados dizem respeito apenas àqueles participantes que foram avaliados em todos os encontros.

A proposta do estudo foi encaminhada e aprovada pelo Comitê de É tica em Pesquisa da U niversidade Federal do Ceará, sob o número de protocolo 58/03, conforme as recomendações referentes às pesquisas desenvolvidas com seres humanos $^{(13)}$. T anto os participantes como os pais ou responsáveis assinaram o termo de consentimento livre e esclarecido, após serem infor mados dos objetivos do estudo.

\section{RESULTAD OS}

Fizeram parte do estudo 92 crianças e 49 adolescentes. A seguir, na T abela 1, tem-se a caracterização dos participantes.

0 fato de se verificar, no grupo avaliado, a presença de $50,4 \%$ crianças e adolescentes do sexo feminino pode ter sido decorrente da ausência no domicílio de diversas pessoas do sexo masculino que, segundo seus familiares, já estavam vinculadas a empregos. G eralmente, na comunidade onde 
0 estudo foi feito, são os homens que começam a trabal har mais cedo, ainda durante a adolescência, tor nando-se, dentro da sociedade, economicamente ativos.

T abela 1 - Caracterização dos sujeitos segundo sexo e grau de parentesco com portador de hipertensão arterial. Fortaleza, CE , 2004-2006.

\begin{tabular}{lcr}
\hline Variáveis & $\mathbf{n}$ & $\%$ \\
\hline G ênero & & \\
$\quad$ M asculino & 70 & 49,6 \\
Feminino & 71 & 50,4 \\
Grau de parentesco & & \\
$\quad$ Filho (a) & 62 & 44,0 \\
N eto (a) & 66 & 46,8 \\
Bisneto (a) & 3 & 2,1 \\
Sobrinho (a) & 10 & 7,1 \\
\hline Total & $\mathbf{1 4 1}$ & $\mathbf{1 0 0 , 0}$ \\
\hline
\end{tabular}

E m relação ao grau de parentesco, ainda como mostra a T abela 1, a maioria das crianças e adolescentes era netos $(46,8 \%)$ ou filhos $(44 \%)$ de portadores de hipertensão arterial.

$\mathrm{Na}$ T abela 2, consta a distribuição dos participantes de acordo com o gênero e valores de pressão arterial nas cinco avaliações.

T abela 2 - Distribuição dos participantes em relação aos valores da pressão arterial sistólica (PAS) e diastólica (PAD) apresentados em cada avaliação de acordo com o gênero. Fortal eza, CE , 2004-2006.

\begin{tabular}{|c|c|c|c|c|c|c|c|}
\hline \multirow{2}{*}{ Avaliação } & \multicolumn{3}{|c|}{ M asculino $(\mathrm{N}=70)$} & \multicolumn{3}{|c|}{ Feminino $(\mathrm{N}=71)$} & \multirow{2}{*}{$\begin{array}{c}\text { Valor } \\
\mathbf{p}\end{array}$} \\
\hline & $M$ & Ep & $\mathrm{Md}$ & $M$ & Ep & $\mathrm{Md}$ & \\
\hline \multicolumn{8}{|l|}{ PAS } \\
\hline $1 \underline{a}$ & 111,61 & 1,281 & 110 & 108,51 & 1,049 & 110 & 0,139 \\
\hline $2 \mathrm{a}$ & 112,80 & 1,651 & 112 & 109,72 & 1,021 & 108 & 0,237 \\
\hline $3 \underline{a}$ & 114,20 & 1,610 & 112 & 109,92 & 0,996 & 108 & 0,071 \\
\hline $4^{\underline{a}}$ & 114,94 & 1,665 & 112 & 11 & 1,071 & 110 & 0,110 \\
\hline $5 \underline{a}$ & 115,29 & 1,684 & 112 & 110,56 & 1,056 & 110 & 0,118 \\
\hline \multicolumn{8}{|l|}{ PAD } \\
\hline $1 \underline{a}$ & 74,09 & 1,098 & 74 & 72,85 & 1,072 & 72 & 0,471 \\
\hline $2^{a}$ & 76,71 & 1,184 & 78 & 74,51 & 0,858 & 76 & 0,135 \\
\hline $3^{a}$ & 77,49 & 1,120 & 78 & 74,73 & 0,848 & 76 & 0,059 \\
\hline $4 \underline{a}$ & 76,26 & 1,246 & 76 & 74,56 & 0,920 & 76 & 0,575 \\
\hline $5 \underline{a}$ & 76,57 & 1,249 & 76 & 74,14 & 0,900 & 74 & 0,292 \\
\hline
\end{tabular}

Legenda: $M=M$ édia, $E \mathrm{p}$ : erro padrão; $M d=M$ ediana.

Conforme se verifica, pela T abela 2 , houve significância marginal na terceira avaliação, tanto da PAS quanto da PAD no referente ao gênero, com $p=0,071$ e 0,059, respectivamente. T ambém, conforme se verifica, as médias de PAS e de PAD foram maiores nos indivíduos do sexo masculino que no feminino em todas as avaliações.

A seguir, na T abela 3, tem-se a distribuição dos participantes segundo a classificação dos percentis da PAS e PAD em todas as avaliações.

T abela 3 - D istribuição dos participantes segundo percentis da pressão arterial sistólica (PAS) e diastólica (PA D) e avaliação. Fortal eza, CE , 20042006.

\begin{tabular}{|c|c|c|c|c|c|}
\hline \multirow{2}{*}{ Percentil } & \multicolumn{5}{|c|}{ Avaliação da pressão arterial ( $\mathrm{N}=141)$} \\
\hline & 19 & 2a & 3 a & $4 \underline{a}$ & 5 a \\
\hline \multicolumn{6}{|l|}{ PAS } \\
\hline Normal & 117 & 115 & 109 & 109 & 109 \\
\hline Limítrofe & 4 & 2 & 14 & 14 & 16 \\
\hline Acima & 20 & 24 & 18 & 18 & 16 \\
\hline \multicolumn{6}{|l|}{ PAD } \\
\hline Normal & 119 & 119 & 89 & 89 & 90 \\
\hline Limítrofe & 6 & 6 & 29 & 29 & 33 \\
\hline Acima & 16 & 16 & 23 & 23 & 18 \\
\hline
\end{tabular}

Como se evidencia pela Tabela 3, o número de indivíduos com percentil de pressão arterial sistólica acima do percentil 90 diminuiu ao longo das cinco aval iações, ao se comparar as últimas três avaliações com a primeira e a segunda. 0 mesmo não ocorreu com os percentis de pressão arterial diastólica.

A o se verificar os percentis tanto de PAS como de PAD com classificação normal, percebe-se que o número de indivíduos diminuiu ao longo das avaliações. Pelo teste de F riedman, comparando a distribuição dos percentis nos vários momentos de avaliação, constatou-se existir diferença significativa $(p<0,001)$ entre a média dos percentis da PAS e PAD nos diversos momentos de aval iação.

Quanto à distribuição das crianças segundo verificações da pressão arterial com alteração dos percentis, verifica-se que ao longo das avaliações, $32,6 \%(30)$ crianças permanecer am sem alter ações dos percentis de pressão arterial nas cinco avaliações; 45,6\% (42) apresentaram alterações a partir da terceira avaliação; 8,7\% (8) mostraram alteração dos percentis somente na última aval iação realizada; 12\% (11) apresentaram alterações dos percentis de pressão arterial em todas as avaliações e 21,7\% (20) evidenciaram alteração dos percentis 
em alguma avaliação, mas na última mostraram percentis normais de pressão arterial (Figura 1).

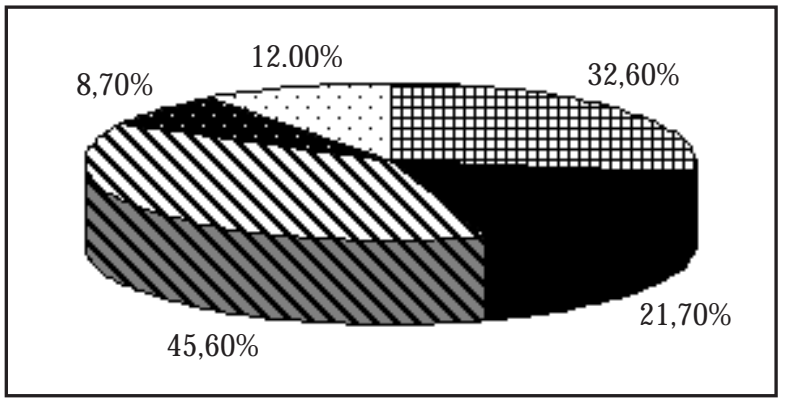

Lengenda: $\boldsymbol{\square}$ nenhuma $\mathbf{a}$ alguma $\mathbf{\nabla}$ a partir da 3ạ $\mathbf{0}$ última $\square$ todas.

Figura 1 - Distribuição das crianças segundo verificações da pressão arterial com alterações dos percentis. Fortal eza, CE , 2004-2006.

Na Figura 2, consta a distribuição dos adolescentes segundo verificações da pressão arterial com alter ação dos percentis. Quando se avaliou os adolescentes, como mostra a figura 2, percebeu-se que $65,3 \%$ (32) permaneceram sem alterações dos percentis de pressão arterial nas avaliações; 16,3\% (8) apresentaram alterações a partir da terceira avaliação; 4,1\% (2) indivíduos mostraram alteração dos percentis somente na última avaliação; $12,2 \%$ (6) revelaram alterações dos percentis de pressão arterial em todas as avaliações e 6,1\% (3) manifestaram alteração dos percentis em alguma avaliação, mas na última mostrar am percentis normais de pressão arterial.

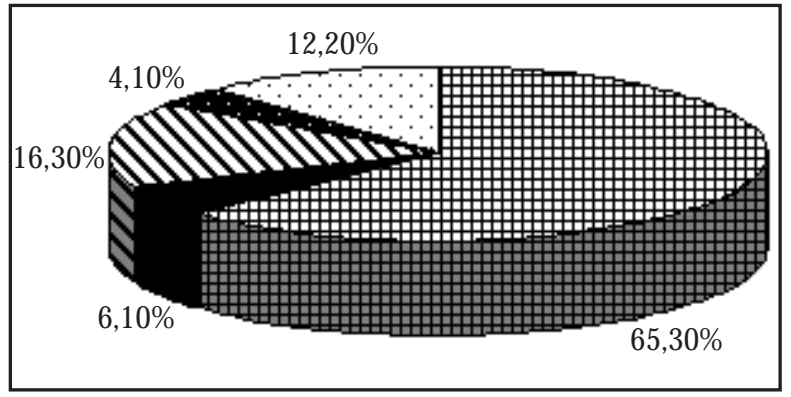

Lengenda: 田nenhuma $\mathbf{a}$ alguma $\mathbf{Q}$ a partir da 3ạ última $\square$ todas.

Figura 2 - Distribuição dos adolescentes segundo verificações da pressão arterial com alterações dos percentis. Fortal eza, CE , 20042006.

\section{DISCUSSÃO}

No que se refere ao gênero dos participantes deste estudo, os resultados evidenciam a mesma tendência de trabalhos similares, com maior freqüência de indivíduos do sexo feminino. E $\mathrm{m}$ um trabal ho epidemiológico foram avaliados 1.253 estudantes entre 7 e 17 anos. D estes, 56,3\% eram do sexo feminino(14).

Quanto à pressão ar terial, conforme este estudo mostrou, as médias de PAS e de PAD foram maiores no sexo masculino e, tanto para participantes do sexo feminino como para o sexo masculino, a média dos valores aumentou com o decorrer do acompanhamento. Este achado corrobora com outra pesquisa que identificou, na população pesquisada, maior número de crianças eadolescentes do sexo masculino com valores maiores, tanto da PAS quanto da PAD, quando comparados às do sexo feminino ${ }^{(15)}$. E m relação às médias obtidas, 0 fato de indivíduos do sexo masculino apresentarem maiores médias de pressões arteriais também foi verificado em outro trabal ho, que avaliou a pressão arterial de adolescentes que exerciam a função de mensageiros na U niver sidade E stadual de Campinas ${ }^{(16)}$.

Outro estudo identificou 97 estudantes com pressão arterial sistólica e/ ou diastólica (média de duas medidas) no percentil > 95, determinando uma prevalência de hipertensão arterial sistêmica de $7,7 \%{ }^{(14)}$.

Como estas pesquisas se caracterizam como transver sais, os valores de pressão arterial são passíveis de modificações ao longo do tempo. Advém, então, mais uma vez, a importância de acompanhamento periódico dos indivíduos em faixas etárias jovens.

Conforme observado, os participantes do estudo em discussão revelaram maior freqüência de alterações da PAS como PAD com o decorrer do tempo de acompanhamento. Este achado difere dos encontrados em um estudo que mostrou diminuição tanto dos val ores correspondentes à PAS como à PAD das crianças e dos adolescentes ao longo do tempo de acompanhamento ${ }^{(15)}$. Contudo, apesar de os dois estudos guardarem semelhanças de contexto e população, no primeiro, foram incluídos escolares com ou sem história familiar de hipertensão arterial e, no estudo ora el aborado, todos os participantes apresentavam história familiar de hipertensão.

No ano de 2009, foi publicado um trabaIho sobre o perfil de pressão arterial e história familiar em crianças escolares. I ncluiu 112 indivíduos entre 6 e 13 anos de idade. D os participantes, sete apresentaram alterações da pressão arterial e todos tinham história familiar para hipertensão arterial, demonstrando que história familiar de hi- 
pertensão esteve associada a maiores valores de pressão arterial nas crianças estudadas ${ }^{(6)}$.

E m indivíduos com al ter ações confirmadas na pressão arterial, é considerada importante uma avaliação mais específica para se traçar o perfil do risco cardiovascular ${ }^{(17)}$. Estes autores mencionam como informações necessárias: a história familiar de doenças cardiovasculares, a presença de diabetes mellitos, dislipidemia ou tabagismo, índice de massa corporal, doença cardiovascular conhecida, estilo sedentário de vida, hábitos de dieta e capacidade de lidar com estresse.

Compete ao enfermeiro abordar aspectos de prevenção e de promoção à saúde, prestar informações, implementar programas educativos e avaliar indivíduos em situação de risco ou que já apresentam alter ações periodicamente, al ém de desenvolver pesquisas sobre a prevenção e o controle da hipertensão arterial|(18).

\section{CONSIDERAÇÕES FINAIS}

Por meio do estudo, foi possível confirmar que crianças e adolescentes podem ter pressões arteriais elevadas, mesmo sem uma aparente causa específica e sem sintomatologia.

Neste estudo, verificaram-se determinados aspectos, tais como: as médias de PAS e PAD, nas cinco avaliações, foram maiores nos indivíduos do sexo masculino que no feminino; o número de indivíduos do grupo com PAS e PAD normais diminuiu ao longo das aval iações, e os percentis de PAD foram os que sofreram maiores alterações com 0 decorrer das avaliações; ademais, 30 (32,6\%) das crianças e $32(65,3 \%)$ dos adolescentes não apresentaram al ter ações dos percentis de pressão ar terial em nenhuma das avaliações.

Conforme se pode inferir, pelos achados, muitos indivíduos (12\%; 12,2\%) não somente mantiveram seus valores e percentis de PAS e PAD elevados, como grande parte $(54,3 \% ; 20,4 \%)$, que não apresentavam inicial mente alterações, passaram a mostrá-las no decorrer do acompanhamento.

É válido ressal tar que, apesar de o M inistério da Saúde, com o estabel ecimento do Programa Saúde do E scolar, que prevêa abordagem da hipertensão em crianças escolares, a ênfase, na prática, ainda são as doenças preval entes na infância (diarréia, desidratação, desnutrição e infecções respiratórias), a saúde bucal e as doenças sexual mente trans- missíveis, estas últimas especificamente para os adolescentes.

D essa forma, salienta-se a importância do acompanhamento destes indivíduos pela equipe de saúde, com vistas a se iniciar medidas preventivas em fase precoce da vida, prevenindo ou mesmo retardando o surgimento da hipertensão na vida adulta ou pel o menos o aparecimento das suas complicações.

As limitações do estudo incluem a perda ocorrida dos participantes e da necessidade da realização de associações dos valores da pressão arterial dos participantes com outras variáveis de risco para o surgimento da hipertensão arterial. Ademais, a comparação de dois grupos de crianças e adolescentes, um com história familiar e o outro sem história familiar de hipertensão arterial, poderia fornecer conclusões mais significativas sobre a influência deste indicador de risco. No entanto, os resultados obtidos ressaltam a necessidade da continuidade de investigação das causas de hipertensão arterial em faixas etárias mais baixas para a implementação de intervenções que visem evitar complicações na vida adulta.

\section{REFERÊ NCIAS}

1 Sociedade Brasileira de Cardiologia, Sociedade Brasileira de Hipertensão, Sociedade Brasileira de $\mathrm{Ne}$ frologia. V Diretrizes Brasileiras de H ipertensão A rterial. Rev Bras Hipert. 2006;13(4):260-312.

2 G uerra RF C, Badía Y V, Rodríguez T RG, U rbina RG. Valores de presión arterial en escolares sanos de una escuela primaria del municipio Cerro. Rev Cubana M ed G en Integr. 2009;25(1):1-8.

3 Araujo T LA, Lopes M VO, M oreira RP, Cavalcante T F, G uedes N G, Silva V M . P ressão arterial de crianças e adolescentes de uma escola pública de Fortal eza - Ceará. A cta Paul E nferm. 2007;20(4):476-82.

4 M ariath $A B$, Grillo LP. Influência do estado nutricional, circunferência da cintura e história familiar de hipertensão sobre a pressão arterial de adolescentes. Rev Ciênc M éd. 2008;17(2):65-74.

5 Ramírez J. Presión normal e hipertensión arterial en niños y adolescentes [ editorial] . A rch Argent Pediatr. 2006;104(3):193-5.

6 A glony $\mathrm{M}$, A rnaiz P, A cevedo M , Barja S, M árquez S, Guzmán $B$, et al. Perfil de presión arterial e historia familiar de hipertensión en niños escolares sanos de 
Santiago de Chile. Rev M ed Chile. 2009;137(1):3945.

7 A raujo T LA, L opes M VO, CavalcanteT F, G uedes NG, M oreira R P, Chaves ES, et al. A nálise de indicadores de risco para hipertensão arterial em crianças e adolescentes. Rev Esc Enferm U SP. 2008;42(1):120-6.

8 V ieira S. Bioestatística: tópicos avançados. Rio de Janeiro: Campos; 2003.

9 Chaves ES. A companhamento de crianças e adolescentes com história familiar de hipertensão arterial [ tese] . Fortal eza: D epartamento de E nfermagem, U niversidade Federal do Ceará; 2007.

10 Chaves ES. Avaliação da pressão arterial em crianças e adolescentes com antecedentes familiares de hipertensão arterial [ dissertação] . Fortal eza: D epartamento de Enfermagem, U niversidade Federal do Ceará; 2004.

11 Rothman K, G reenland S. M oder n epidemiology. $2^{2 a}$ ed. P hiladelphia: Lippincott \& Raven; 1998.

12 Perloff D, Grim C, Flack J, Frohlich DE, Hill M, $M C D$ onald $M$, et al. Human blood pressure determination by sphygmomanometry: part I. Circulation. 1993;88:2460-70.

13 M inistério da Saúde (BR), Conselho N acional de Saúde. N ormas de pesquisa envolvendo seres humanos: Res. CNS 196/ 96. Bioética. 1996;4(2 Supl):15-25.
14 Silva M AM, Rivera IR, Ferraz M RM T, Pinheiro $A J T$, Alves SW S, M oura AA, et al. Prevalência de fatores de risco cardiovascular em crianças e adolescentes da rede de ensino da cidade de M aceió. Arq Bras Cardiol. 2005;84(5):387-92.

15 M oreira R P. A companhamento da pressão arterial de crianças e adolescentes: estudo em uma escola de Fortal eza - Ceará [ monografia] . Fortal eza: D epartamento de Enfermagem, U niversidade Federal do Ceará; 2006.

16 Spinella C, L amas J LT. F atores associados à hipertensão arterial e níveis pressóricos encontrados entre adolescentes trabal hadores. Rev E sc E nferm U SP. 2007;41(2):196-204.

17 Vagaonescu T, Phillips RA. Testes iniciais rotineiros para diagnóstico e estratificaçãode risco do paciente com hipertensão. In: Weber M A. H ipertensão. Rio de Janeiro: G uanabara Koogan; 2003. p. 8791.

$18 \mathrm{G}$ asperin $\mathrm{D}$, Fenster seifer L M . As modificações do estilo de vida para hipertensos. Rev $\mathrm{G}$ aúcha $\mathrm{E}$ nferm. 2006;27(3):372-8.

\section{AG RADECIMENT OS}

A o Consel ho $\mathrm{N}$ acional de $\mathrm{D}$ esenvolvimento Científico eT ecnológico (CN Pq), pelo apoio ao Projeto Ações de Cuidado em Saúde Cardiovascular, no 474475/ 2007-7.

\section{Endereço da autora / Dirección del autor / Author's address:}

Emilia Soares Chaves

Rua São H ipólito, 1100, casa 9, A lagadiço N ovo 60830-700, Fortaleza, CE

E-mail: emiliasoareschaves@yahoo.com.br
Recebido em: 06/ 11/ 2009

A provado em: 09/ 03/ 2010 